\title{
Flow resistance reduction of coal water slurry through gas phase addition
}

\author{
Jolanta Robak ${ }^{1, a}$, and Karina Ignasiak ${ }^{1}$ \\ ${ }^{1}$ Institute for Chemical Processing of Coal, 41-803 Zabrze, Zamkowa 1 St., Poland
}

\begin{abstract}
One of the main advantages of coal water slurry fuel (CWS) is a physical form that allows, among others, their transfer by pipelines over long distances. For this form of transport actions towards reducing the flow resistance of the transmitted medium are important. One of the treatments leading to reduction in the flow resistance of suspensions is to introduce gas into the stream of flowing slurry. The goal of that action is to either loosen the structure of densely packed grains or increase the velocity of the suspension. The paper presents the flow resistance of CWS in a horizontal pipeline and the effect of addition of the gas phase on the resistance level. The investigation was carried out with the use of a research stand enabling to measure the flow resistance of the multiphase/multicomponent systems. The measured diameter and length of sections were respectively: 0.03 and $2 \mathrm{~m}$. The coal-water slurries (based on steam coals) with concentration of dry coal in the range of 51 do $60 \%$ obtained by wet milling in a drum mill were used. During the tests, the following parameters were measured: slurry flow rate, air flow rate, temperature and pressure difference in inlet and outlet of the measured section. The volume flow rate of slurry fuel was in the range of 30 to $110 \mathrm{dm}^{3} / \mathrm{min}$ while the volume flow rate of air was from 0.15 to $4 \mathrm{~m}^{3} / \mathrm{h}$. Based on the obtained results, the slurry flow resistance as a function of the flow rate and share of introduced air was evaluated. The performed research allowed for assessment of flow resistance reduction condition and to determine the pipe flow curves for different temperatures. It was found that the effect of reducing the flow resistance of the coal slurry by introducing gas into the flow tube depended on the volumetric flow rate, and thus the linear velocity of the slurry. Under the experimental condition, this effect only occurred at low flow rates $\left(30-50 \mathrm{dm}^{3} / \mathrm{min}\right)$ and low temperature of the suspension. The share of air needed to flow resistance reduction was in the range of 0.02 to $0.04 \%$ by volume.
\end{abstract}

${ }^{\text {a }}$ Corresponding author: office@ichpw.pl 